\title{
Infección atípica y neurorretinitis por Bartonella henselae en una unidad de hospitalización pediátrica: comunicación de tres casos
}

\author{
Bartonella henselae with atypical infection and neuroretinitis in a pediatric unit: report of \\ three cases
}

Regina Pérez , Alexandra Fischman², María José Poblete², Cecilia Vizcaya' ${ }^{1}$ Cecilia Perret' ${ }^{1}$ Cristián García ${ }^{3}$, Aldo Muñoz y Nicole Le Corre

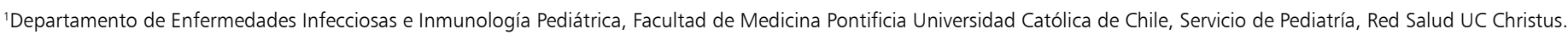
Santiago, Chile.

${ }^{2}$ Escuela de Medicina, Pontificia Universidad Católica de Chile. Santiago, Chile.

${ }^{3}$ Departamento de Radiología, Facultad de Medicina, Pontificia Universidad Católica de Chile. Santiago, Chile.

${ }^{4}$ Departamento de Oftalmología, Facultad de Medicina, Pontificia Universidad Católica de Chile. Santiago, Chile.
\end{abstract}

Los autores declaran no tener conflictos de interés en la publicación de este artículo.

Sin fuentes de financiamiento.

Recibido: 7 de octubre de 2019 / Aceptado: 12 de julio de 2020

\section{Resumen}

Bartonella henselae es el agente etiológico de la enfermedad por arañazo de gato (EAG), infección endémica en Chile. Típicamente se presenta como una linfadenopatía regional autolimitada y menos frecuentemente con compromiso sistémico y manifestaciones extraganglionares: en hígado, bazo, hueso, ojo, entre otros. Se presentan tres casos de infección atípica por Bartonella henselae en las que se evidenció compromiso ocular, manifestado como una neurorretinitis. Esta revisión destaca la importancia de la búsqueda activa de complicaciones oculares en pacientes con compromiso sistémico por Bartonella henselae, implicando un cambio en el tratamiento y pronóstico de la enfermedad.

Palabras clave: Bartonella henselae; enfermedad por arañazo de gato; neurorretinitis; fondo de ojo.

\section{Introducción}

L a enfermedad por arañazo de gato (EAG) es una patología infecciosa, producida por la bacteria Bartonella henselae. Corresponde a bacilos gramnegativos intracelulares, fastidiosos, aeróbicos y oxidasa-negativos ${ }^{1}$. El gato es su principal reservorio y cumple un rol fundamental en la transmisión de la infección a los humanos, quienes la adquieren a través del arañazo o mordida de gatos

\begin{abstract}
Bartonella henselae is cat scratch disease's etiological agent, which is considered an endemic infection in Chile. It typically presents as a self-limited regional lymphadenopathy and less frequently with systemic involvement and extranodal or atypical manifestations: hepatosplenic, ocular or musculoskeletal involvement, among others. We present three cases of atypical cat scratch disease with ocular compromise, as neurorretinitis. This review highlights the importance of the active search for ocular complications in patients with disseminated cat scratch disease, leading to possible change in treatment and prognosis of the disease.

Keywords: Bartonella henselae; cat scratch disease; neurorretinitis; ocular fundus.
\end{abstract}


a tres semanas después del contacto con gatos, y que se acompaña generalmente por fiebre baja, compromiso del estado general y anorexia ${ }^{4}$. La piel en torno a la lesión puede presentar eritema y aumento del calor local (lesión de inoculación $)^{5}$. Esta forma de presentación llamada “típica" se observa en $88-89 \%$ de los casos. Por otra parte, la enfermedad puede presentarse de manera "atípica", desarrollando manifestaciones extraganglionares como consecuencia de la diseminación hematógena de la bacteria. Algunas de estas formas extraganglionares son el síndrome febril prolongado, el compromiso hepatoesplénico, óseo, neurológico, ocular, cardiológico, entre otros $^{4}$. Se postula que estas diferentes formas de presentación serían consecuencia de la virulencia de distintas cepas de Bartonella spp. y de la respuesta inmune inducida por la bacteria en el hospedero ${ }^{4,6,7}$.

El compromiso ocular se evidencia en 5 al $10 \%$ de los pacientes con EAG, siendo la forma de presentación más frecuente el síndrome oculoglandular de Parinaud ${ }^{1,8}$. Si bien, la neurorretinitis es una presentación menos habitual, es la complicación del segmento posterior ocular más frecuente de la infección por Bartonella spp. Es una forma de neuropatía óptica inflamatoria con un característico exudado estrellado en el área macular ${ }^{1,8}$. La enfermedad se manifiesta por pérdida visual indolora subaguda y autolimitada, en general unilateral ${ }^{9}$. Bartonella henselae es la etiología identificada más común de neurorretinitis a nivel mundial ${ }^{10,11}$.

El objetivo de esta revisión es destacar el potencial compromiso ocular de la EAG a través de la comunicación de tres casos clínicos de pacientes pediátricos con una infección sistémica por $B$. henselae documentada serológicamente, en los que se evidenció una neurorretinitis.

\section{Casos clínicos}

\section{Caso 1}

Escolar de sexo femenino 7 años, previamente sana, que consultó por un cuadro de tres semanas de evolución de fiebre sobre $38^{\circ} \mathrm{C}$, diaforesis, dolor cervical y dorsal con signos inflamatorios locales sobre las apófisis espinosas e impotencia funcional, asociado a compromiso del estado general, anorexia y baja de peso. Refería contacto con animales (perros, gatos y gallinas), sin antecedentes de alimentos de riesgo, ni viajes. Al examen físico destacaba dolor a la palpación de las apófisis espinosas dorsales, sin otros hallazgos. En los exámenes de laboratorio de ingreso se observó un hemograma con hemoglobina $11,4 \mathrm{~g} / \mathrm{dl}$, leucocitos 10.000 céls $/ \mathrm{mm}^{3}, 54 \%$ polimorfonucleares, plaquetas 530.000 céls $/ \mathrm{mm}^{3}$, PCR 4,24 mg/dl $(\mathrm{VN}<0,5)$, VHS $83 \mathrm{~mm} / \mathrm{h}$, perfíl bioquímico normal, orina completa con microhematuria (48 eritrocitos/uL). Dentro del estudio etiológico se realizaron hemocultivos, urocultivo, aglutinación para Brucella abortus/melitensis, serología
Brucella canis, ELISA para VIH y QuantiFERON-TB ${ }^{\circledR}$ todos negativos, obteniéndose una $\operatorname{IgG} B$. henselae positiva (título $1 / 1024)(\mathrm{VN}<1 / 256)$. Se completó el estudio con una ecografía abdominal que mostró múltiples lesiones focales hepato-esplénicas compatibles con granulomas y una radiografía de tórax con un nódulo pulmonar de $18 \mathrm{~mm}$ en el tercio medio del hemitórax izquierdo. Se realizó un cintigrama óseo donde se observaron áreas de mayor captación en la cuarta y novena costillas, vértebras T7-T10-L3 y acetábulo derecho, hallazgos concordantes con múltiples focos de osteomielitis. Se realizó un fondo de ojo (FO) que mostró un edema de papila bilateral. Se inició tratamiento sistémico tri-asociado con cotrimoxazol, rifampicina oral y ciprofloxacina intravenosa por siete días. La paciente evolucionó satisfactoriamente, afebril desde el ingreso y sin dolor. Completó seis semanas de terapia antimicrobiana por vía oral con el mismo esquema.

\section{Caso 2}

Escolar de sexo femenino de 9 años de edad, con antecedentes de trastorno de conducta alimentaria y talla baja en estudio. Consultó a un oftalmólogo por una pérdida visual progresiva de una semana de evolución, inicialmente con un escotoma central evolucionando hacia el compromiso completo del campo visual del ojo derecho. Presentó, además, coriza, tos escasa y compromiso del estado general, sin fiebre ni lesiones cutáneas. Dentro de los antecedentes epidemiológicos refería el contacto con animales (perros, gatos y aves de compañía), sin contacto con enfermos, alimentos de riesgo ni viajes. Al examen ocular se observó una intensa disminución de la visión del ojo derecho, destacando una visión mejor corregida de 0,4 p y la presencia de edema de papila y una estrella macular con abundante exudado lipídico en dicha área (Figura 1). Dado lo característico de la presentación clí-

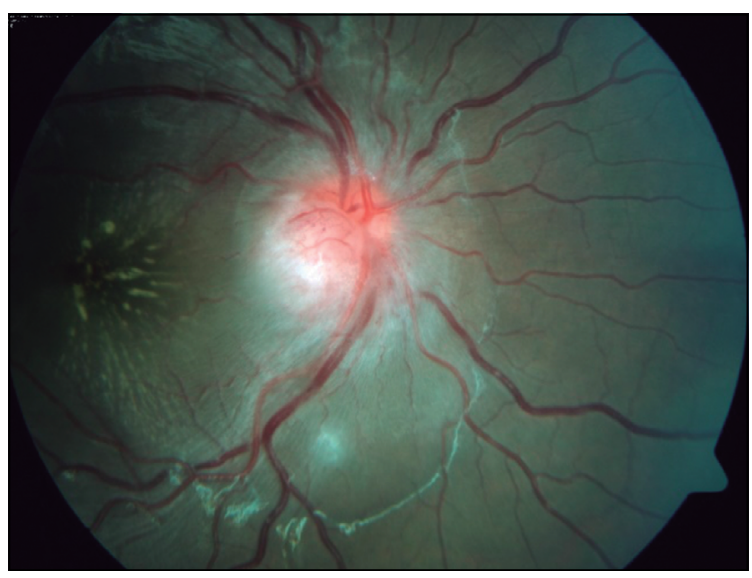

Figura 1. Fondo de ojo derecho: Edema intenso de papila y exudados lipídicos en el área foveal, compatible con una neurorretinitis, probablemente secundaria a una infección por Bartonella spp. 
nica se diagnosticó una neurorretinitis. Por este motivo se derivó para su hospitalización y estudio. Al examen físico del ingreso se objetivó una pérdida de la visión central del ojo derecho y la presencia de rasguños en el brazo ipsilateral, el resto del examen no presentaba hallazgos patológicos. De los exámenes de laboratorio destacaban leucocitos 6.850 céls $/ \mathrm{mm}^{3}$ (neutrófilos $51,4 \%$, linfocitos $34,7 \%$ y eosinófilos $5,3 \%$ ), PCR $2,03 \mathrm{mg} / \mathrm{dl}(\mathrm{VN}<0,5)$, VHS $39 \mathrm{~mm} / \mathrm{h}$, pruebas hepáticas normales. En el estudio etiológico infeccioso se obtuvo una IgM negativa e IgG positiva en $230 \mathrm{UI} / \mathrm{mL}$ para Toxoplasma gondii, IgG negativa para Toxocara canis y un RPR negativo. La serología IgG para $B$. henselae fue positiva, con título de $1 / 1024$, lo que apoyó el diagnóstico de una neurorretinitis por $B$. henselae. Resultaron positivos también los anticuerpos anti nucleares Hep 2 1/80, con ANCA y anti DNA negativos y complemento $\mathrm{C} 3-\mathrm{C} 4$ normales. La ecografía abdominal mostró múltiples imágenes nodulares hipoecogénicas en el bazo, de hasta $3 \mathrm{~mm}$, compatibles con granulomas. Se inició tratamiento con doxiciclina y rifampicina por seis semanas. A las tres semanas de iniciado el tratamiento, se realizó un campo visual computarizado de control, que evidenció un escotoma centro-cecal no recuperado (Figura 2) y exudados lipídicos densos en el área foveal. Se ajustó el tratamiento a ciprofloxacina asociado a rifampicina, hasta completar siete semanas de terapia. En el seguimien-

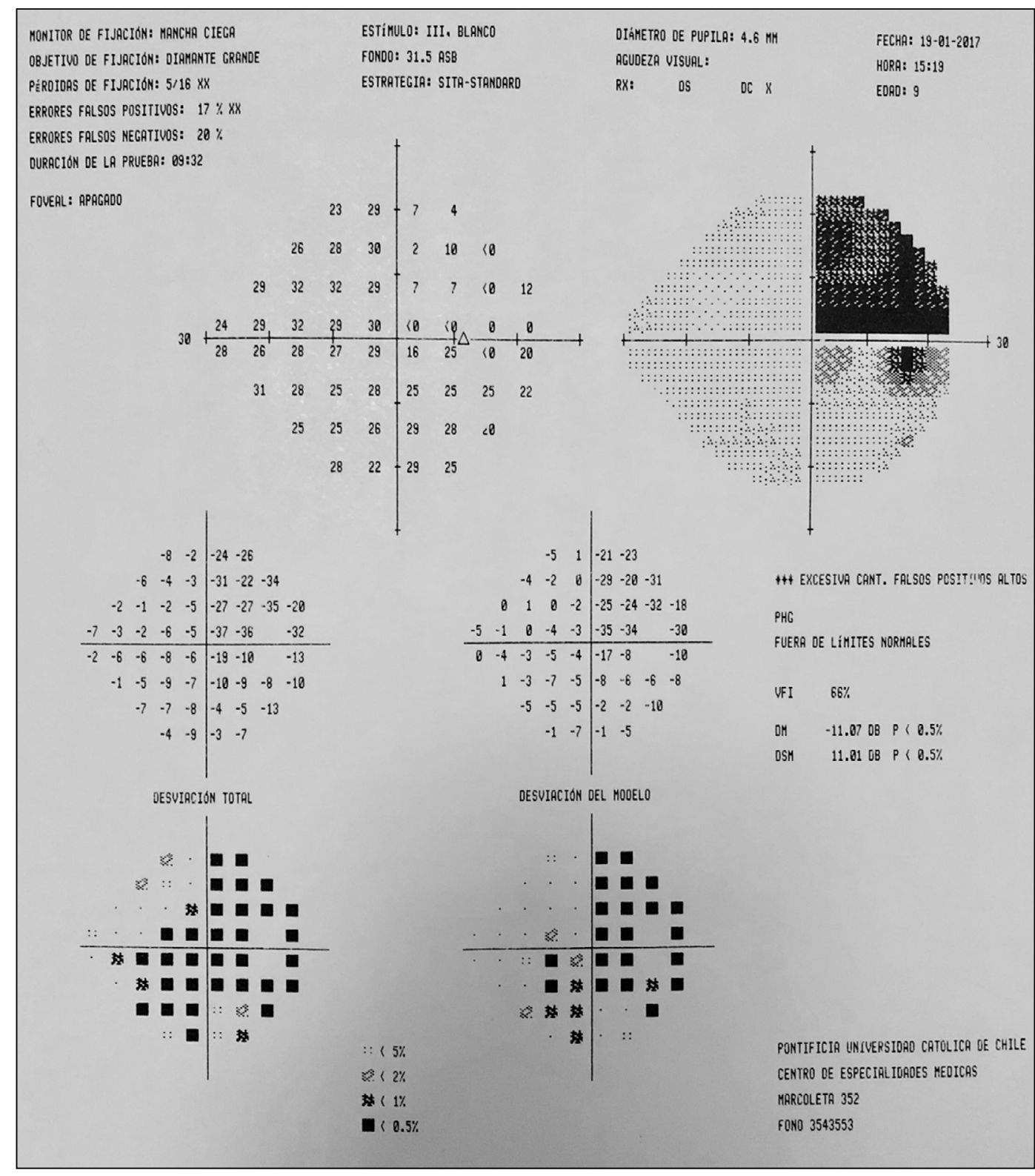

Figura 2. Campo visual 24/2. Destaca la presencia de escotoma centro cecal del ojo afectado. 


\section{-}

Figura 3. Fondo de ojo izquierdo. Edema intenso de papila asociado a dilataciones vasculares y pliegues de la membrana limitante interna concéntricos al nervio óptico. En el área macular exudados lipídicos en forma de estrella macular.

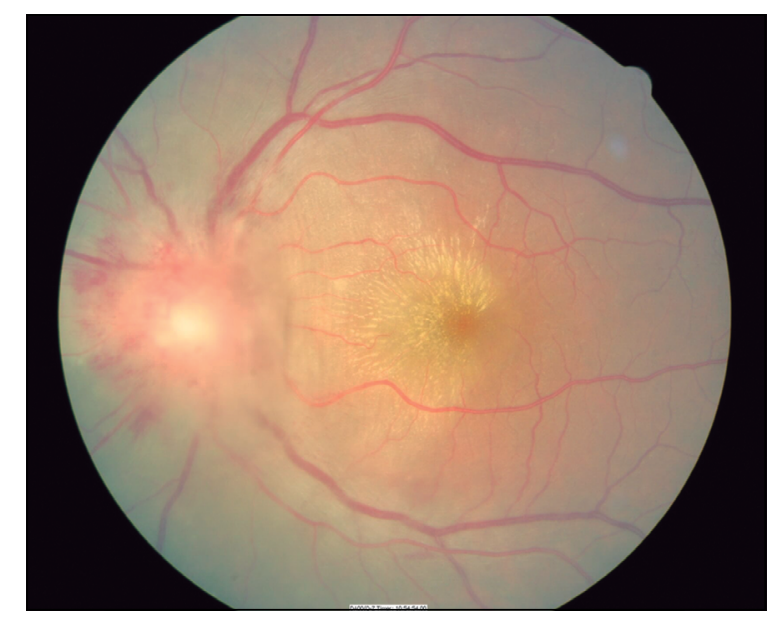

to oftalmológico se evidenció la resolución del edema de papila y mejoría de su agudeza visual (mejor corregida hasta $0,8 \mathrm{p}$ a los seis meses), pero con persistencia de algunos exudados lipídicos en el área foveal. En control a los 18 meses posterior al cuadro inicial, la paciente presentaba una visión de $1,0 \mathrm{p}$ con corrección óptica y desaparición de los exudados lipídicos; sin embargo, se observaba una sutil alteración residual a nivel del epitelio pigmentario retinal.

\section{Caso 3}

Escolar de sexo femenino de 12 años de edad, sin antecedentes relevantes. Consultó en el servicio de urgencias por un cuadro de seis días de evolución de fiebre hasta $40^{\circ} \mathrm{C}$ y calofríos, asociado a cefalea intermitente y dolor tóraco-lumbar. Dentro de los antecedentes epidemiológicos refirió contacto con animales (gatos y perros), sin contactos con enfermos, viajes, fármacos ni alimentos de riesgo. Al examen físico se encontraba afebril y con dolor en región sacroilíaca a la palpación, sin dolor a la movilización. De los exámenes de laboratorio de ingreso destacan leucocitos 8.210 céls $/ \mathrm{mm}^{3}$ (segmentados $61 \%$, linfocitos 31\%), PCR 7,35 mg/dl (VN <0,5), VHS 98 $\mathrm{mm} / \mathrm{h}$, perfil bioquímico y orina completa sin alteraciones. En el estudio etiológico se obtuvo un panel viral respiratorio molecular, hemocultivos y urocultivo negativos, IgM para parvovirus negativa, IgG positiva CMV, con IgM indeterminada, IgG $B$. henselae positiva con título $>1 / 1024$. Se realizó una radiografía de tórax que mostró un leve infiltrado bilateral, la ecografía abdominal normal y un cintigrama óseo con una captación en el codo derecho y fisis de peroné distal izquierdo. La resonancia magnética de codo derecho mostró una imagen compatible con una adenopatía epitroclear reactiva. Se realizó un FO que evidenció la presencia de un intenso edema de papila, con dilataciones vasculares sobre la cabeza del nervio óptico, asociado a un desprendimiento de retina seroso en el área peri-papilar y pliegues concéntricos de la membrana limitante interna. Estas alteraciones del nervio óptico, asociadas a exudados lipídicos en forma de estrella macular, fueron hallazgos compatibles con una neurorretinitis (Figura 3). Se inició tratamiento triasociado con gentamicina intravenosa por $48 \mathrm{~h}$, y posteriormente doxiciclina y rifampicina vía oral hasta completar seis semanas. Durante el seguimiento se evidenció una evolución clínica favorable, con un hemograma y VHS normal, y un FO con regresión completa de las alteraciones al mes de iniciada la terapia.

\section{Discusión}

El ojo es el órgano no linfático más comúnmente afectado en la EAG atípica ${ }^{5}$. El compromiso ocular puede producirse por vía hematógena o por inoculación de la bacteria en la conjuntiva, siendo sus presentaciones variadas y de diversa gravedad ${ }^{12}$. El síndrome oculoglandular de Parinaud compromete principalmente la conjuntiva y los ganglios linfáticos regionales y es la manifestación ocular más frecuente, presentándose en cerca de $5 \%$ de los $\operatorname{casos}^{13}$. Se manifiesta con epífora, hiperemia conjuntival y sensación de cuerpo extraño. Al examen físico destacan granulomas conjuntivales y la presencia de adenopatías preauriculares, submandibulares o cervicales $^{8}$. Sin embargo, puede haber también compromiso del segmento posterior del ojo manifestándose como una neurorretinitis, neuritis/papilitis óptica, oclusiones vasculares, retinitis interna aguda multifocal o puntos blancos retino-coroídeos, vasculitis retinal, retinitis, coroiditis focal, desprendimiento retinal seroso, uveítis intermedia, entre otros ${ }^{14-16}$.

La neurorretinitis es la complicación del segmento posterior más frecuente de la EAG, presentándose en $1-2 \%$ de los pacientes con infección por $B$. henselae ${ }^{13}$. Corresponde a una forma de neuropatía óptica inflamatoria, caracterizada por disminución de agudeza visual uni o bilateral, debido a edema del disco óptico asociado en ocasiones a un desprendimiento retinal seroso del área macular y aparición de exudado en patrón estrellado (parcial o completo) alrededor de la fóvea ${ }^{17}$. El hallazgo más característico en el FO es el edema del disco óptico, a menudo acompañado de líquido subretinal peripapilar, que generalmente precede a la formación de una estrella macular, parcial o completa en una a tres semanas ${ }^{18}$. Sin embargo, algunos pacientes nunca forman la estrella macular ${ }^{19}$, mientras que otros desarrollan una inflamación masiva del disco con exudados subretinianos e intrarretinales generalizados ${ }^{20}$. Por lo tanto, frente a un cuadro de edema papilar en un niño hay que realizar un seguimiento clínico y oftalmológico estricto, dentro del cual se debe 
efectuar un FO y campo visual seriado, para evaluar una posible progresión a estrella macular ${ }^{21}$. El compromiso del nervio óptico en forma de lesión angiomatosa peri-papilar o como granuloma de disco óptico es poco frecuente ${ }^{15}$. Algunas manifestaciones adicionales que podrían asociarse son hemorragias intrarretinales, uveítis anterior o intermedia, coroiditis o retinitis focal o multifocal e incluso oclusiones arteriales y venosas retinales ${ }^{22,23}$. Exámenes no invasivos, que permiten confirmar este diagnóstico, son la angiografía con fluoresceína ocular y la tomografía de coherencia óptica (OCT, por su sigla en inglés). El hallazgo principal en la angiografía con fluoresceína es la filtración del contraste a nivel del nervio óptico. Otros hallazgos incluyen la angiomatosis peripapilar y el desprendimiento de retina seroso peripapilar ${ }^{20}$.

La causa más frecuente de neurorretinitis es la infección por $B$. henselae, existiendo evidencia serológica en aproximadamente dos tercios de los $\operatorname{casos}^{10}$. Dentro de los diagnósticos diferenciales a considerar se encuentran la sífilis, tuberculosis, histoplasmosis, enfermedad de Lyme, toxoplasmosis, toxocariasis, leptospirosis, sarcoidosis, hipertensión maligna, diabetes mellitus e hipertensión endocraneana ${ }^{8,24}$.

El diagnóstico de infección sistémica por $B$. henselae puede realizarse por métodos directos e indirectos, incluyendo la serología, cultivos, histología, inmunohistoquímica y biología molecular ${ }^{5}$. Las pruebas serológicas corresponden al método más utilizado en Chile para la confirmación diagnóstica de la infección. Puede realizarse mediante inmunofluorescencia indirecta (IFI) o ELISA, con distintos valores de sensibilidad y especificidad $^{24}$. La detección de IgG mediante inmunofluorescencia (IFI) con un corte $>1 / 64$, presenta una sensibilidad de $88-100 \%$ y una especificidad de $92-98 \%$, siendo considerada el estándar de oro ${ }^{25,26}$. Dado que en Chile existe una seroprevalencia de al menos $13 \%$ en la población pediátrica se utiliza un punto de corte mayor (1/256) para la IgG por IFI $^{3}$ para hacer diagnóstico de enfermedad y distinguirlo de la infección pasada. Por otra parte, la IgM anti- $B$. henselae por IF no cumple aún los requisitos para reemplazar a la IgG como examen diagnóstico por su baja sensibilidad ${ }^{25}$. Si la medición de IgG resulta negativa y existe una alta sospecha clínica de infección por $B$. henselae, se debe repetir la IgG una a dos semanas después ${ }^{27}$. Cabe destacar que títulos elevados de IgG refuerzan el diagnóstico de EAG, tal como se observó en los casos presentados. Los cultivos pueden realizarse en sangre o tejidos, no obstante, resulta laborioso, ya que $B$. henselae es una bacteria fastidiosa que requiere incubación en medios enriquecidos para su óptimo desarrollo y es de lento crecimiento (9 a 40 días $)^{5}$. También es posible realizar el diagnóstico mediante estudio inmunohistoquímico en muestras de tejidos de biopsia con tinción de Warthin Starry (que evidencia granulomas con la bacteria en su interior) $)^{1,4,28}$. Con respecto a técnicas de biología molecular, la RPC constituye un método diagnóstico rápido, inequívoco y útil principalmente cuando la serología resulta negativa o no concluyente ${ }^{29}$. Se dispone de una RPC específica y universal. Las muestras para su análisis se pueden obtener de diversos tejidos, según el compromiso clínico (más frecuentemente de linfadenopatías). No obstante, la RPC se realiza ocasionalmente en la práctica clínica ${ }^{30}$.

No todos los pacientes con compromiso ocular presentan síntomas clínicos evidentes. Las manifestaciones oculares en general aparecen después de una a cuatro semanas de los síntomas sistémicos, pudiendo también presentarse en ausencia de éstos, como ocurrió en el segundo caso clínico ${ }^{19,22}$. Considerando que no todos los pacientes con compromiso ocular manifiestan síntomas clínicamente apreciables, y que 1 a $2 \%$ de los pacientes con infección sistémica por $B$. henselae presentan neurorretinitis, es fundamental mantener un alto índice de sospecha, y por lo tanto, realizar FO a todos los pacientes con EAG atípica, como ocurrió en los casos clínicos 1 y 3 expuestos. Por otra parte, frente a todo paciente con signos clínicos de neurorretinitis en el FO, debe solicitarse serología de $B$. henselae (como se realizó en el caso 2), considerando que los hallazgos del examen no siempre orientan a la etiología causal y que esta bacteria representa la principal etiología infecciosa descrita ${ }^{10}$. Una vez que se comprueba que la neurorretinitis es secundaria a infección por $B$. henselae, se considera una EAG atípica y dado que ésta implica una fisiopatología hematógena, siempre se debe descartar el compromiso hepatoesplénico con una ecografía abdominal, ya que en la mayoría de los casos son asintomáticos, como se observó en los casos clínicos 1 y 2. En estos casos la ecografía puede mostrar imágenes nódulares hipoecogénicas pequeñas en cuantía variable en el bazo, con o sin compromiso asociado del hígado. La cintigrafìa ósea y la RM son de utilidad cuando se sospecha compromiso osteoarticular, como ocurrió en dos de los casos descritos.

Con respecto a la terapia de la EAG, la indicación de tratamiento antimicrobiano en los pacientes inmunocompetentes con infección por $B$. henselae típica es controversial, dado que la evidencia disponible que sustenta el uso de azitromicina proviene de sólo un estudio aleatorizado y con beneficio marginal ${ }^{31}$. Sin embargo, cuando se trata de una EAG atípica, la tendencia es a usar tratamiento antibacteriano. La neurorretinitis por EAG en general tiene un pronóstico favorable, siendo la mayoría de los casos autolimitados ${ }^{32}$. No obstante, algunos pacientes evolucionan con secuelas mínimas, entre las que se incluyen defectos del campo visual, disminución leve de la agudeza visual, visión de colores alterada o sensibilidad al contraste disminuida ${ }^{23}$. El tratamiento antimicrobiano se sugiere para evitar estos riesgos. Se 


\section{- Grci caso Clinico}

ha observado también, que los pacientes que presentan deterioro de la agudeza visual, dado frecuentemente a la presencia de neurorretinitis en el FO pueden beneficiarse de la terapia ${ }^{30}$. La recomendación para el tratamiento de la neurorretinitis es la terapia combinada con rifampicina y doxiciclina en pacientes mayores de 8 años y rifampicina con azitromicina o cotrimoxazol para pacientes de 7 años o menores ${ }^{8,33,34}$. En el caso de los pacientes presentados, dos de ellos recibieron rifampicina con doxiciclina, con buena respuesta. Sin embargo, la paciente del caso 2 cambió de terapia por falta de respuesta, asociándose ciprofloxacina a su tratamiento, al igual que la paciente del primer caso. En la literatura especializada se describe que el género Bartonella es sensible a las fluoroquinolonas, pero aún faltan estudios que demuestren su superioridad respecto a otros antibacterianos ${ }^{32}$. No se ha determinado la duración óptima de terapia para pacientes inmunocompetentes con EAG complicada; sin embargo, se sugiere completar un período mínimo de cuatro a seis semanas $^{11,33}$. Los corticosteroides se han considerado parte del tratamiento, en particular en pacientes con pérdida moderada a severa de la agudeza visual, pero faltan aún estudios que apoyen su uso ${ }^{1,30}$.

Los casos clínicos expuestos demuestran la importancia de realizar sistemáticamente un examen de FO cuando la EAG se presenta en forma atípica, en particular con evidencia de diseminación hematógena. Asimismo, en presencia de neurorretinitis se debe descartar el compromiso de otros órganos frecuentemente afectados en la infección por $B$. henselae. Si bien los hallazgos del FO son característicos, un título alto de IgG específica refuerza el diagnóstico etiológico. La neurorretinitis es una manifestación poco frecuente de la EAG, pero se debe realizar una búsqueda activa de esta patología para conocer su real incidencia en la población pediátrica.

\section{Referencias bibliográficas}

1.- Florin T A, Zaoutis T E, Zaoutis L B. Beyond cat scratch disease: widening spectrum of Bartonella henselae infection. Pediatrics 2008; 121: e1413-25. doi: 10.1542/peds.2007-1897.

2.- Ferres M, Abarca K, Godoy P, García P, Palavecino E, Mendez G, et al. Presence of Bartonella henselae in cats: natural reservoir quantification and human exposition risk of this zoonoses in Chile. Rev Med Chile 2005; 133 : 1465-71. doi: /S0034-98872005001200008.

3.- Ferres G M, Abarca V K, Prado D P, Montecinos P L, Navarrete C M, Vial C P. Prevalence of Bartonella henselae antibodies in Chilean children, adolescents and veterinary workers. Rev Med Chile 2006; 134: 863-7. doi: /S0034-98872006000700009.

4.- Gandhi T N, Slater L N, Welch D F, Koehler J E. Chapter 236-Bartonella, Including CatScratch Disease. Bennett JE DR, Blaser MJ. Mandell, Douglas and Bennett's Principles and Practice of Infectious Diseases. 8th ed. Philadelphia; 2015, Vol 2, pags 2649-63.e4.

5.- Massei F, Gori L, Macchia P, Maggiore G. The expanded spectrum of bartonellosis in children. Infect Dis Clin North Am 2005; 19: 691-711. doi: 10.1016/j.idc.2005.06.001.

6.- Harms A, Dehio C. Intruders below the radar: molecular pathogenesis of Bartonella spp. Clin Microbiol Rev 2012; 25: 42-78. doi: 10.1128/ CMR.05009-11.

7.- McGill S L, Regnery R L, Karem K L. Characterization of human immunoglobulin (Ig) isotype and $\operatorname{IgG}$ subclass response to Bartonella henselae infection. Infect Immun 1998; 66: 5915-20.
8.- Cunningham E T, Koehler J E. Ocular bartonellosis. Am J Ophthalmol 2000; 130: 340-9. doi: 10.1016/s0002-9394(00)00573-0.

9.- $\mathrm{Hu}$ V, Dong B, MacFarlane A. Visual loss after cat scratch. $J$ R Soc Med 2005; 98: 28-9. doi: 10.1258/jrsm.98.1.28.

10.- Suhler E B, Lauer A K, Rosenbaum J T. Prevalence of serologic evidence of cat scratch disease in patients with neuroretinitis. Ophthalmology 2000; 107: 871-6. doi: 10.1016/ s0161-6420(00)00002-6.

11.- Abdelhakim A, Rasool N. Neuroretinitis: a review. Curr Opin Ophthalmol 2018; 29: 514-9. doi: 10.1097/ICU.0000000000000527.

12.- Ormerod L D, Dailey J P. Ocular manifestations of cat-scratch disease. Curr Opin Ophthalmol 1999; 10: 209-16. doi: 10.1097/00055735199906000-00010.

13.- Carithers H A. Cat-scratch disease. An overview based on a study of 1,200 patients. Am J Dis Child 1985; 139: 1124-33. doi: 10.1001/archpedi.1985.02140130062031.

14.- Tager M, Jahnsen J, Mediavilla M, Burgos R. Ocular bartonellosis: Report of three clinical cases. Rev Chilena Infectol 2008; 25: 58-63. doi: /S0716-10182008000100012.

15.- Aziz H A, Plesec T P, Sabella C, Udayasankar U K, Singh A D. Cat Scratch Disease: Expanded spectrum. Ocul Oncol Pathol 2016; 2: 246-50. doi: 10.1159/000447063.

16.- Biancardi A L, Curi A L. Cat-scratch disease. Ocul Immunol Inflamm 2014; 22: 148-54. doi: 10.3109/09273948.2013.833631.

17.- Purvin V, Sundaram S, Kawasaki A. Neuroretinitis: review of the literature and new observations. J Neuroophthalmol 2011; 31: 5868. doi: 10.1097/WNO.0b013e31820cf78a.
18.- Pepose J S H G, Wilhelmus K R. Ocular Infection and Immunity. St. Louis: Mosby Year Book; 1996. p. 601-8.

19.- Wade N K, Levi L, Jones M R, Bhisitkul R, Fine L, Cunningham E T, Jr. Optic disk edema associated with peripapillary serous retinal detachment: an early sign of systemic Bartonella henselae infection. Am J Ophthalmol 2000; 130: 327-34. doi: 10.1016/ S0002-9394(00)00599-7.

20.- Cunningham E T Jr, McDonald H R, Schatz H, Johnson R N, Ai E, Grand M G. Inflammatory mass of the optic nerve head associated with systemic Bartonella henselae infection. Arch Ophthalmol 1997; 115: 1596-7. doi: 10.1016/ S0002-9394(00)00599-7.

21.- Del Valle D V. Neurorretinitis por Bartonella henselae: presentación de un caso y revisión de la literatura. Rev Mex Oftalmol 2015; 90: 23541. doi: 10.1016/j.mexof.2015.06.007.

22.- Ormerod L D, Skolnick K A, Menosky M M, Pavan P R, Pon D M. Retinal and choroidal manifestations of cat-scratch disease. Ophthalmology 1998; 105: 1024-31. doi: 10.1016/S0161-6420(98)96003-1.

23.- Reed J B, Scales D K, Wong M T, Lattuada C P Jr, Dolan M J, Schwab I R. Bartonella henselae neuroretinitis in cat scratch disease. Diagnosis, management, and sequelae. Ophthalmology 1998; 105: 459-66. doi: 10.1016/S01616420(98)93028-7.

24.- Arce N. Neurorretinitis asociada a infección por Bartonella henselae: reporte de un caso clínico y revisión de la literatura. Rev Hosp Clin Univ Chile 2017; 28 (12-7).

25.- Abarca K, Winter M, Marsac D, Palma C, Contreras A M, Ferres M. Accuracy and 


\section{Caso Clínico}

diagnostic utility of IgM in Bartonella henselae infections. Rev Chilena Infectol 2013; 30: 125 8. doi: 10.4067/S0716-10182013000200001.

26.- English R. Cat-scratch disease. Pediatr Rev 2006; 27: 123-8; quiz 128. doi: 10.1542/pir.274-123.

27.- Pérez G J, Munita S J, Araos B R, López G J, Stevenson A R, González A P, et al. Cat scratch disease associated neuroretinitis: clinical report and review of the literature. Rev Chilena Infectol 2010; 27: 417-22. doi: 10.4067/S071610182010000600008 .

28.- Gouriet F, Lepidi H, Habib G, Collart F, Raoult D. From cat scratch disease to endocarditis, the possible natural history of Bartonella henselae infection. BMC Infect Dis 2007; 7 (30). doi: 10.1186/1471-2334-7-30.

29.- Fukushima A, Yasuoka M, Tsukahara M, Ueno $\mathrm{H}$. A case of cat scratch disease neuroretinitis confirmed by polymerase chain reaction. Jpn J Ophthalmol 2003; 47: 405-8. doi: 10.1016/ s0021-5155(03)00074-1.

30.- Habot-Wilner Z, Trivizki O, Goldstein M, Kesler A, Shulman S, Horowitz J, et al. Catscratch disease: ocular manifestations and treatment outcome. Acta Ophthalmol 2018; 96 : e524-e32. doi: 10.1111/aos.13684.

31.- Bass J W, Freitas B C, Freitas A D, Sisler C L, Chan D S, Vincent J M, et al. Prospective randomized double blind placebo-controlled evaluation of azithromycin for treatment of catscratch disease. Pediatr Infect Dis J 1998; 17:
447-52. doi: 10.1097/00006454-19980600000002 .

32.- Rolain J M, Brouqui P, Koehler J E, Maguina C, Dolan M J, Raoult D. Recommendations for treatment of human infections caused by Bartonella species. Antimicrob Agents Chemother 2004; 48: 1921-33. doi: 10.1128/ AAC.48.6.1921-1933.2004

33.- Durá Travé T, Yoldi Petri ME, Lavilla Oiz A, Molins Castiella T. Neuroretinitis in cat-scratch disease. An Pediatr (Barc) 2010; 72: 290-1. doi: 10.1016/j.anpedi.2009.12.005.

34.- Amer R, Tugal-Tutkun I. Ophthalmic manifestations of Bartonella infection. Curr Opin Ophthalmol 2017; 28: 607-12. doi: 10.1097/ICU.0000000000000419. 